\section{A controlled trial of the effect of topical glyceryl trinitrate on skin blood flow and skin elasticity in scleroderma}

It has been suggested that in systemic sclerosis (SSc) the primary fault is in the microcirculation. ${ }^{1}$ If this were so, then topical glyceryl trinitrate (GTN) might favourably influence skin blood flow and elasticity in areas of sclerodermatous skin change.

Ten patients (three men, seven women; median age 48 years, range 24-70) fulfilling the American Rheumatism Association criteria for $\mathrm{SSc}^{2}$ were recruited into a doubleblind placebo-controlled clinical trial. All had forearm skin involvement and the median disease duration was 2.5 years, range seven months to 18 years. The extent of forearm skin involvement varied between patients. Most had mild skin thickening (involved, but skin could be pinched) and only one had classical 'hidebound' skin.

For four weeks each patient applied topical GTN $(2 \%)$ three times daily to a designated area of one forearm, and a lanolin-based placebo ointment to the corresponding area of the other. Careful instructions and a demonstration of how much ointment to apply were given and the patient wore gloves to minimise absorption from other sites. Elasticity (using a Cutech Extensometer), skin blood flow (by the laser Doppler technique using a Perimed PF3 instrument) and skin temperature (using a Heimann KT41 Bolometer) were measured at both sites at base-line, one hour post initial ointment application, and after two and then four weeks of treatment. The third and fourth assessments (at two and four weeks respectively) were made three hours after ointment application. Coefficients of variance (median and range) are $7.6 \%$ (1.7-22) for elasticity measurements, $42 \%$ (6-56) for laser Doppler readings and $2 \%$ $(0 \cdot 6-4)$ for bolometry.

Baseline and post-treatment results within each group (GTN and placebo treated) were compared using a Signed rank test. Changes from baseline were compared between groups using a Mann-Whitney U-test.

Two patients withdrew from the study because of adverse effects of the trial treatment. One withdrew after three days, complaining of a stinging sensation in both arms following the application of the trial treatment. The other withdrew after the two week visit, complaining of discomfort in what proved to be the arm treated with GTN, and headaches. Data were therefore only available from these patients for the first two and three assessments respectively. Otherwise all the patients completed all assessments, with the exception of one patient who, due to computer malfunction, did not have a laser Doppler measurement at her second (one hour post-first application) assessment.

Results for elasticity, laser Doppler, and bolometry assessments are shown in the table. With the exception of a statistically significant difference between the bolometry readings of the GTN treated group at baseline and one hour, there were no significant changes over time in any of the three parameters measured in either the GTN or placebo treated forearms. Nor were there any significant differences in these changes between the two groups.

Elasticity, blood flow and temperature measurements in topical GTN and placebo-treated forearms

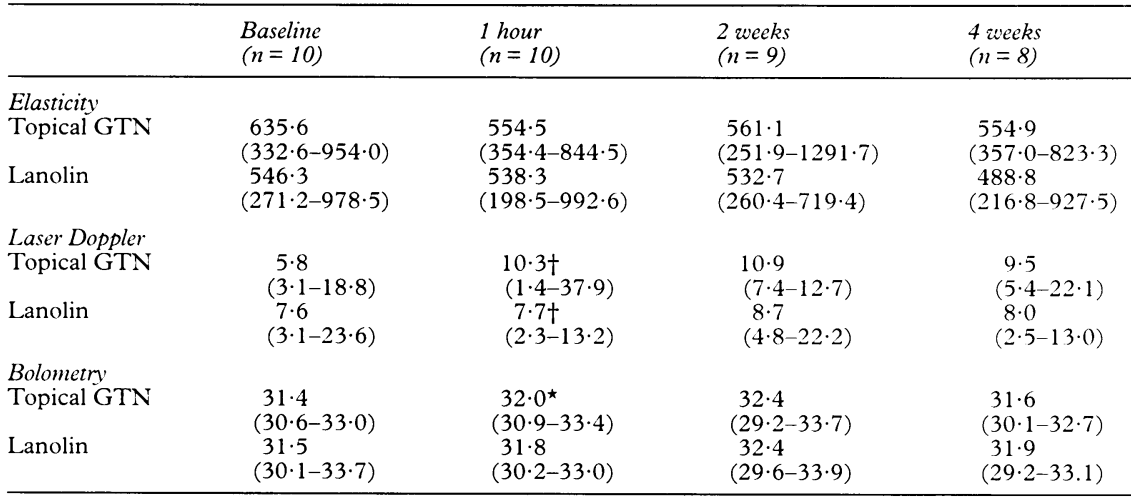

${ }^{*} \mathrm{p}<0.05$ versus baseline

$+n=9$

Results are median (range)

Regarding ointment utilisation, this varied considerably between patients but there was a tendency for patients to use similar amounts on each arm. Similar amounts of GTN and placebo ointments were used by the nine patients completing the first two week period as follows: median amount of GTN consumed $4.6 \mathrm{~g}$, range 1.4 to $8.1 \mathrm{~g}$, median amount of placebo ointment used $3 \cdot 2 \mathrm{~g}$, range $1 \cdot 1$ to $7 \cdot 3 \mathrm{~g}$.

The application of topical GTN over a four week period therefore had no effect on skin elasticity or blood flow detectable by the methods used. Possible explanations for these negative findings include the importance of factors other than the microcirculation in the pathogenesis of SSc, the short time interval studied, and dermal blood vessel wall changes which were already irreversible in this patient group at the time of study.

It has been suggested that topical GTN is helpful in the management of secondary Raynaud's, in combination with oral methyldopa or guanethidine. ${ }^{3}$ Coppock et al demonstrated a reduction in cold-induced vasospasm after topical GTN in patients with secondary Raynaud's. ${ }^{4}$ Our study, however, did not examine the circulation of the hand but skin thickening and skin blood flow of the forearm.

Any benefit from topically applied GTN is likely to arise from a combination of local and systemic effects. Our study was useful in demonstrating that in most patients the treatment was well tolerated, and also that ointment application (either placebo or GTN) did not in itself distort skin elasticity. Further studies should be longer term and include patients with early disease in whom dermal microvascular changes are likely still to be reversible.

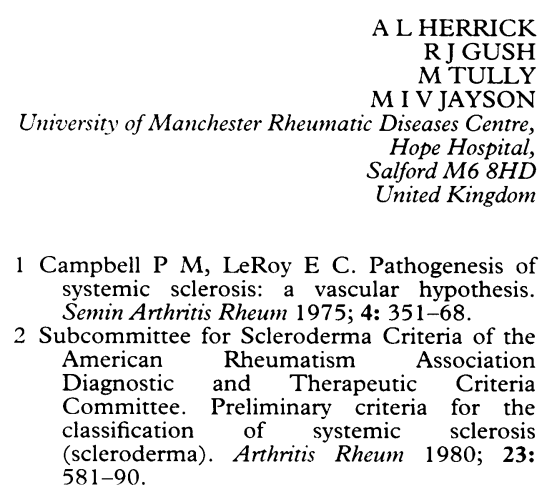

3 Franks A G. Topical glyceryl trinitrate as adjunctive treatment in Raynaud's disease. Lancet 1982; i: 76-7.

4 Coppock J S, Hardman J M, Bacon P A Woods K L, Kendall M J. Objective relief of vasospasm by glyceryl trinitrate in secondary Raynaud's phenomenon. Postgrad Med $\mathcal{f}$ 1986; 62: 15-8.

\section{Osteoporosis and Ehlers- Danlos syndrome}

The changes in bone mineral content of patients with Ehlers-Danlos syndrome (EDS) are still not wholly defined. Although well described in diseases of the same group (osteogenesis imperfecta, homocystinuria), osteoporosis is seldom consistently referred to in association with EDS

Prockop suggests that the mutations altering the sequence of the $\mathrm{N}$-terminal extremity of the procollagen, as is the case with some groups of EDS, would not cause a diminution of the bone mass. ${ }^{2}$ However, some studies appear to indicate that in EDS there is an alteration in the normal bone metabolism, finding an increase in the frequency of vertebral fractures and ultrastructural changes in the collagen fibrils. It also seems that there is a lower absorption of tetracycline in the bone tissue of patients with type I EDS. ${ }^{3}$

We recently assessed, using dual energy $x$ ray absorptiometry (Hologic QDR-1000), four of six patients with type I EDS observed in the outpatients clinic of the Rheumatology Unit of the Hospital Militar Principal (the two patients not included were children aged four and seven years), three male and one female patient, with ages ranging from 16-25 years. None of the patients presented other significative risk factors for low bone mass and none had clinical or radiological evidence of vertebral crush fractures. Data obtained were compared to our reference data, composed of healthy Portuguese students, male and female (military and young female workers of military units). Each year of life of our reference group is composed of 30 individuals of each sex (ex: $16 \mathrm{y}-30 \mathrm{male}$ and 30 female). The BMD values found proved to be, at the level of the lumbar spine, persistently below 1 standard deviation of the average for sex and age. These differences were minimal or non existent for the femur neck (see table). 